\title{
Special Lattice of Rough Algebras
}

\author{
Yonghong Liu \\ School of Automation, Wuhan University of Technology, Wuhan, China \\ E-mail: hylinin@163.com \\ Received October 14, 2011; revised November 16, 2011; accepted November 24, 2011
}

\begin{abstract}
This paper deals with the study of the special lattices of rough algebras. We discussed the basic properties such as the rough distributive lattice; the rough modular lattice and the rough semi-modular lattice etc., some results of lattice are generalized in this paper. The modular lattice of rough algebraic structure can provide academic base and proofs to analyze the coverage question and the reduction question in information system.
\end{abstract}

Keywords: Lattice, Rough Distributive Lattice, Rough Modular Lattice, Rough Semi-Modular Lattice

\section{Introduction}

It is generally known that the order and the partial order set theory were widely applied in the discrete mathematics and fuzzy mathematics. In algebraic theories about the notion of lattice as both profound and sweeping.

The rough set was introduced by Pawlak in 1982 [1]. The lattice to characterize rough set is an important task [2-6]. Actually, we can use a lattice model to represent different information flow policies and play an important role in Boolean algebra. Obviously, the coverage problem and the reductions problem are two problems of the cores in information system of lattice relation, which boosts the development of lattice theory. We give several special lattices of rough algebras that we discuss in this article; for instance, we prove that a lattice is necessary and sufficient condition of the rough semi-modular lattice.

We will now describe a lattice definition and then we introduce rough approximation spaces. The main contents are as the following:

Definition 1.1. [7] Let $L$ is a set. Define the meet $(\wedge)$ and join $(\vee)$ operations by

$$
\begin{aligned}
& x \wedge y=\operatorname{glb}(x, y), \\
& x \vee y=\operatorname{lub}(x, y) .
\end{aligned}
$$

The following properties hold for all elements $x, y, z \in L$.

1) commutative laws:

$$
x \wedge y=y \wedge x \text { and } x \vee y=y \vee x .
$$

2) associative laws:

$$
\begin{aligned}
& (x \wedge y) \wedge z=x \wedge(y \wedge z) \text { and } \\
& (x \vee y) \vee z=x \vee(y \vee z)
\end{aligned}
$$

3) absorption laws:

$$
x \wedge(x \vee y)=x \text { and } x \vee(x \wedge y)=x .
$$

4) idempotent laws:

$$
x \wedge x=x \text { and } x \vee x=x .
$$

A lattice is an algebra structure $\langle L, \vee, \wedge\rangle$ that has two binary composition $\wedge$ and $\vee$, it satisfies the abovementioned condition 1), 2), 3) and 4).

Definition 1.2. [7] Let $L$ is a lattice, if for any $x, y, z \in L$.

$$
\begin{aligned}
& \text { 1) } x \wedge(y \vee z)=(x \wedge y) \vee(x \wedge z) \text {, or } \\
& \text { 2) } x \vee(y \wedge z)=(x \vee y) \wedge(x \vee z) .
\end{aligned}
$$

Therefore, $L$ is called a distributive lattice.

Definition 1.3. [7] A distributive lattice is called a modular lattice.

Theorem 1.1. [7] Let $L$ is modular lattice, then $L$ is called a semi-modular lattice.

Definition 1.4. [8] Assume that $U$ is a finite and nonempty set with the universe, $R \subseteq U \times U$ denote a binary relation on $U$. Let $(U, R)$ is an approximation spaces. Define $\mathbb{R}=\{(\underline{R}, \bar{R}) \mid R \subseteq U\}$ is a rough approximation spaces. $\underline{R}$ and $\bar{R}$ are referred to as the lower and upper approximation operators respectively.

Theorem 1.2. [8] Let $(U, R)$ be an approximation spaces. Then algebra $\langle\mathbb{R}, \vee, \wedge\rangle$ is a complete distributive lattice.

\section{Main Results}

Definition 2.1. Let $(U, R)$ be an approximation spaces. For all $x, y \in R$. If $\underline{R}(x)=\underline{R}(y)$, then the rough $x$ and $y$ 
are called lower rough equal. The notation $x=y$ denotes that $x$ and $y$ are lower rough equal. If $\bar{R}(x)=\bar{R}(y)$, then the rough $x$ and $y$ are called upper rough equal. The notation $x \simeq y$ denotes that $x$ and $y$ are upper rough equal. If $x \approx y$ and $x \simeq y$, then the rough $x$ and $y$ are called rough equal. The notation $x \approx y$ denotes that $x$ and $y$ are rough equal.

Definition 2.2. Let $\vee x_{t}, \wedge x_{t} \in \mathbb{R}$, and $S$ be a unary operation. We use the notation

$$
\begin{aligned}
& \underset{t \in T}{\vee} X_{t} \approx \underset{t \in T}{\vee} \underline{X}_{t} \vee S\left(\underset{t \in T}{\vee} \bar{x}_{t}\right), \\
& \wedge \hat{x}_{t \in T} \approx \underset{t \in T}{\wedge} \underline{x}_{t} \vee S\left(\underset{t \in T}{\wedge} \bar{X}_{t}\right) .
\end{aligned}
$$

The definition represents that the rough union and rough intersection (where $T$ is an index set).

Definition 2.3. Let algebra $\langle\mathbb{R}, \vee, \wedge\rangle$ be a rough lattice, if for any $x, y, z \in R$, satisfying

$$
x \preceq y \Rightarrow x \vee(z \wedge y) \approx(x \vee z) \wedge y,
$$

Therefore, $R$ is a rough modular lattice.

Theorem 2.1. The rough distributive lattice $\langle\mathbb{R}, \vee, \wedge\rangle$ is rough modular lattice.

Proof. Suppose that $R$ is a rough distributive lattice, if for any $x, y, z \in R, x \preceq y$, then

$$
\begin{aligned}
& x \vee(z \wedge y) \approx(\underline{x} \vee(\underline{y} \wedge \underline{z})) \vee S(\bar{x} \vee(\bar{y} \wedge \bar{z})) \\
& \approx((\underline{x \vee \underline{y}}) \wedge(\underline{x} \vee \underline{z})) \vee S((\bar{x} \vee \bar{y}) \wedge(\bar{x} \vee \bar{z})) \\
& \approx(x \vee y \wedge \underline{x \vee z}) \vee S(\overline{x \vee y} \wedge \overline{x \vee z}) \\
& \approx(x \vee y \wedge x \vee z) \vee S(\overline{x \vee y \wedge x \vee z}) \\
& \approx(x \vee y) \wedge(x \vee z) \\
& \approx(x \vee z) \wedge y .
\end{aligned}
$$

Hence the $\langle\mathbb{R}, \vee, \wedge\rangle$ is rough modular lattice.

Theorem 2.2. The rough modular lattice $\langle\mathbb{R}, \vee, \wedge\rangle$ is rough distributive lattice, if and only if for any $x, y, z \in R$, the following formulas hold:

$$
\begin{aligned}
& (x \wedge y) \vee(y \wedge z) \vee(z \wedge x) \\
& \quad \approx(x \vee y) \wedge(y \vee z) \wedge(z \vee x) .
\end{aligned}
$$

Proof. Necessity:

If for any $x, y, z \in R$, then

$$
\begin{aligned}
& (x \wedge y) \vee(y \wedge z) \vee(z \wedge x) \\
& \approx(((x \wedge y) \vee y) \wedge((x \vee y) \vee z)) \vee(z \wedge x) \\
& (\text { distributive laws }) \\
& \approx(y \wedge(x \vee z) \wedge(y \vee z)) \vee(z \wedge x) \\
& (\text { absorption laws and distributive laws) } \\
& \approx(y \vee z) \wedge(y \vee x) \wedge(x \vee z \vee z) \wedge(x \vee z \vee x) \\
& \wedge(y \vee z \vee z) \wedge(y \vee z \vee x)
\end{aligned}
$$

(distributive laws)

$$
\approx(y \vee z) \wedge(x \vee y) \wedge(z \vee x) \wedge(x \vee y \vee z)
$$

(idempotent laws and commutative laws)

$$
\approx(x \vee y) \wedge(y \vee z) \wedge(z \vee x) .
$$

Sufficiency:

If for any $x, y, z \in R$, then

$$
\begin{aligned}
x \wedge(y \vee z) & \approx x \wedge(x \vee z) \wedge(y \vee z) \\
& \approx x \wedge(x \vee y) \wedge(x \vee z) \wedge(y \vee z) \\
& \approx x \wedge((x \vee y) \wedge(y \vee z) \wedge(z \vee x)) \\
& \approx x \wedge((x \wedge y) \vee(y \wedge z) \vee(z \wedge x)) \\
& \approx((x \wedge y) \vee(y \wedge z) \vee(z \wedge x))) \wedge x
\end{aligned}
$$

Because $x \wedge y \preceq x$, and since the rough modular laws. We see that

$$
\begin{aligned}
& ((x \wedge y) \vee((y \wedge z) \vee(z \vee x))) \wedge x \\
& \quad \approx(x \wedge y) \vee(((y \wedge z) \vee(z \wedge x)) \wedge x) .
\end{aligned}
$$

Because $z \wedge x \preceq X$, and since the rough modular laws. It follows that

$$
(x \wedge y) \vee(z \wedge x) \vee(y \wedge z \wedge x) \approx(x \vee y) \vee(x \wedge z) .
$$

We conclude that

$$
x \wedge(y \vee z) \approx(x \vee y) \vee(x \wedge z) .
$$

Show that $\langle\mathbb{R}, \vee, \wedge\rangle$ is the rough distributive lattice.

Theorem 2.3. A necessary and sufficient condition that rough lattice $R$ is a rough modular lattice, for any $x, y \in R, x \preceq y$ and $z \in R$, we have

$$
x \vee z \approx y \vee z, x \wedge z \approx y \wedge z \text {, then } x \approx y .
$$

Proof. Necessity:

Sufficiency:

$$
\begin{aligned}
x & \approx x \vee(x \wedge z) \\
& \approx x \vee(y \wedge z) \\
& \approx x \vee(z \wedge y) \\
& \approx(x \vee z) \wedge y \\
& \approx(y \vee z) \wedge y \approx y .
\end{aligned}
$$

Let $x \preceq y$. To show that $z$, we thus have

$$
x \vee(z \wedge y) \approx(x \vee z) \wedge y .
$$

We shall prove that the two laws:

$$
\begin{aligned}
& \text { 1) }(x \vee(z \wedge y)) \wedge z \approx((x \vee z) \wedge y) \wedge z, \\
& \text { 2) }(x \vee(z \wedge y)) \vee z \approx((x \vee z) \wedge y) \vee z .
\end{aligned}
$$

To prove 1). In fact,

$$
\begin{aligned}
& ((x \vee z) \wedge y) \wedge z \approx((x \vee z) \wedge z) \wedge y \approx z \wedge y, \\
& (x \vee(z \wedge y)) \wedge z \\
& \quad \preceq(y \vee(z \wedge y)) \wedge z \approx y \wedge z \approx z \wedge y, \text { and } \\
& y \wedge z \approx z \wedge y \approx(y \wedge z) \wedge z \preceq(x \vee(z \wedge y)) \wedge z,
\end{aligned}
$$

thus 


$$
((x \vee z) \wedge y) \wedge z \approx z \wedge y
$$

For part 2),

$$
(x \vee(z \wedge y)) \vee z \approx x \vee((z \wedge y) \vee z) \approx x \vee z .
$$

and

$$
((x \vee z) \wedge y) \vee z \preceq(x \vee z) \vee z \approx x \vee z .
$$

Since $x \preceq y$, we conclude that $x \wedge y \approx x$, then

$$
x \vee z \approx(x \wedge y) \vee z \preceq((x \vee z) \wedge y) \vee z,
$$

thus

$$
((x \vee z) \wedge y) \vee z \approx x \vee z,
$$

which proves 2).

Definition 2.4. The $R$ is called a rough semi-modular lattice denotes that $\bar{x}$ is coverage of $\bar{x} \wedge \underline{y}$, and $\underline{y}$ is also coverage of $\bar{x} \wedge \underline{y}$, then $\bar{x} \vee \underline{y}$ is coverage of $\bar{x}$ and it is also coverage of $y$.

Theorem 2.4. If $R$ is a rough modular lattice, then $R$ is a rough semi-modular lattice.

Proof. Let $\bar{x}$ be coverage of $\bar{x} \wedge y$ and let $\underline{y}$ is also coverage of $\bar{x} \wedge \underline{y}$, if for every $f \bar{\in} R$, and

$$
\bar{x} \preceq f<\bar{x} \vee \underline{y},
$$

which proves that $f \approx \bar{x}$, whence $\bar{x} \vee \underline{y}$ is coverage of $\bar{x}$. In fact,

$$
\bar{x} \preceq f<\bar{x} \vee \underline{y},
$$

we have

$$
\bar{x} \wedge \underline{y} \preceq f \wedge \underline{y} \preceq(\bar{x} \vee \underline{y}) \wedge \underline{y} \approx \underline{y},
$$

but $f \wedge y \neq y$, if not, $\bar{x} \preceq f$ and $y \preceq f$. This leads to the contradiction $\bar{x} \vee y \preceq f$. We see that $y$ is coverage of $\bar{x} \wedge \underline{y}$, so that

$$
\bar{x} \wedge \underline{y} \approx f \wedge \underline{y}
$$

thus

$$
f \approx f \wedge(\underline{y} \vee \bar{x}) \approx(f \vee \underline{y}) \vee \bar{x} \approx(\bar{x} \wedge \underline{y}) \vee \bar{x} \approx \bar{x} .
$$

Similarly, $\bar{x} \vee y$ is coverage of $y$.

Theorem 2.5. Ássume that rough semi-modular lattice $R_{1}, R_{2}, \cdots, R_{n}$. Then the Cartesian product $R \approx R_{1} \times R_{2} \times \cdots \times R_{n}$ is a rough semi-modular lattice.

Proof. Let $\bar{x} \approx\left(\bar{x}_{1}, \bar{x}_{2}, \cdots, \bar{x}_{n}\right)$ and $\underline{y} \approx\left(y_{1}, y_{2}, \cdots, \underline{y}_{n}\right) \in R, R \approx R_{1} \times R_{2} \times \cdots \times R_{n}$, and let $\bar{x}$, $\underline{y}$ are coverage of $\bar{x} \wedge \underline{y} \approx\left(z_{1}, z_{2}, \cdots, z_{n}\right)$, if there exists $i$, then $\bar{x}_{i}$ is coverage of $z_{i}$, and let $k \neq i$, we have $z_{k} \approx \bar{x}_{k}$. If there exists $j$, then $\underline{y}_{j}$ is coverage of $z_{j}$, and let $k \neq j$, we have $z_{k} \approx y_{k}$. If $i \neq j$, then $\bar{x} \vee y \approx\left(t_{1}, t_{2}, \cdots, t_{n}\right)$, where $t_{i} \approx \bar{x}_{i}, t_{j} \approx y_{j}, t_{k} \approx z_{k}$ $(k \not \bar{i}, j)$.

Here, $\bar{x} \vee y$ is coverage of $\bar{x}$, it is also coverage of $y$. If $i \approx j$, then $\bar{x} \vee y \approx\left(u_{1}, u_{2}, \cdots, u_{n}\right)$, where

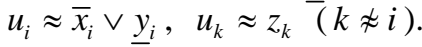

Because $R_{i}$ be a rough semi-modular lattice, hence $u_{i}$ is coverage of $\bar{x}_{i}$, and it is also coverage of $y_{i}$. Therefore, $\bar{x} \vee y$ is not only coverage of $\bar{x}$, but also coverage of $y$.

Corollary ${ }^{-}$2.1. Let $(U, R)$ be an approximation spaces. Suppose that $X$ is a nonempty set, $X \subseteq U$, and $R$ be a set of equivalent relation, then $R$ is a rough semi-modular lattice based on the inclusion relation.

\section{References}

[1] Z. Pawlak, "Rough Sets,” International Journal of Computer and Information Sciences, Vol. 11, No. 5, 1982, pp. 341-356. doi:10.1007/BF01001956

[2] M. Novotny and Z. Pawlak, "Characterization of Rough Top Equalities and Rough Bottom Equalities,” Bulletin of the Polish Academy of Sciences. Mathematics, Vol. 33, No. 1-2, 1985, pp. 91-97.

[3] D. Dubois and H. Prade, "Rough Fuzzy Sets and Fuzzy Rough Sets,” International Journal of General Systems, Vol. 17, No. 2-3, 1990, pp. 191-209. doi:10.1080/03081079008935107

[4] Y. H. Liu, "Lattice to Characterizing Rough Set," Pattern Recognition and Artificial Intelligence, Vol. 16, No. 2, 2003, pp. 174-177.

[5] W. Q. Liu and C. X. Wu, “The Approximation Operator on F-Lattice,” Acta Mathematical Seneca, Vol. 46, No. 6, 2003, pp. 1163-1170.

[6] G. L. Liu, “The Lattice Properties of Rough Set Quotient Spaces,” Computer Engineering \& Science, Vol. 26, No. 12, 2004, pp. 82-90.

[7] D. C. Sheng, “Abstract Algebra,” Science Press, Beijing, 2001, pp. 114-127.

[8] W. X. Zhang, W. Z. Wu, J. Y. Liang and D. Y. Li, "Rough Sets: Theory and Methods," Science Press, Beijing, 2001, pp. 72-77. 